\title{
Intracellular and Intercellular RNA Trafficking in Plants
}

\author{
Biao Ding \\ Department of Plant Cellular and Molecular Biology and Plant Biotechnology Center, \\ The Ohio State University, Columbus, Ohio
}

RNA is rapidly emerging at the forefront of biological investigations, as a result of the discovery of its many unexpected regulatory functions in gene expression, organismal development and host-pathogen interactions. In plants, ample evidence suggest the existence of an RNA trafficking machinery that selectively transports RNAs from cell to cell to regulate gene expression at the organismal level, and such machinery is used by infectious RNAs such as viruses to spread infection. We are interested in the question of how an RNA template is transcribed in the nucleus and is then selected for trafficking across different cellular boundaries. To address this question, we use as our model system a small, noncoding and infectious RNA, the potato spindle tuber viroid (PSTVd), which replicates in the nucleus and infects a plant systemically, and we employ a combination of microscopic, molecular and genetic approaches.

Using cryosections, fluorescence in situ hybridization and confocal microscopy, we found that PSTVd RNAs of sense- and antisense polarities produced during replication are preferentially localized in separate subnuclear compartments as a result of selective trafficking. Furthermore, our studies revealed distinct subnuclear structures associated with viroid RNA localization, implicating their general significance in RNA metabolism in a plant nucleus. Our observations establish a cellular basis for further investigations into the molecular and cellular mechanisms underlying the replication of an infectious RNA template. They also suggest the existence of endogenous mechanisms to separate or co-localize cellular RNAs of sense and antisense polarities as a means of gene regulation.

Using a combination of cryosections, in situ hybridization and genetic analyses, we have obtained evidence that trafficking of the viroid RNA across different cellular boundaries in opposite directions is regulated by distinct RNA motifs. This finding not only serves as a basis to further dissect the various viroid RNA motifs that mediate trafficking throughout a whole plant to establish infection, but also has broad implications in investigating how the trafficking of viral and cellular RNAs across different cellular boundaries is controlled. Finally, studies of this nature are providing new insights into plant structure and function relationships. 